\title{
FOUR-BODY RST GENERAL NUCLEAR WAVEFUNCTIONS AND MATRIX ELEMENTS
}

\author{
IRFAN CHAUDHARY and PETER HAGELSTEIN \\ Research Laboratory of Electronics \\ Massachusetts Institute of Technology \\ Cambridge, MA 02139,USA \\ E-mail: irfanc@mit.edu,plh@mit.edu
}

\begin{abstract}
The inclusion of phonon exchange in a nuclear reaction is accomplished most easily when the associated matrix elements are written explicitly as a function of the spatial coordinates. We report on the wavefunctions and matrix elements for the special case of a $T=0$ 4-body deuteron-deuteron fusion reaction.
\end{abstract}

\section{Introduction}

Physicists have long assumed that the dynamics of nuclear reactions in condensed matter proceeds fast enough that successful approximations can be obtained using a vacuum approximation. Such an approach has been widely successful for many decades in the case of nuclei that have been accelerated. The many experimental claims for anomalous effects in metal deuterides, such as excess heat, helium, low-level fusion, and fast alpha emission, suggest that the use of the vacuum approximation may not be universally applicable. We have been interested in the development of models in which the vacuum approximation is not made, and in which the nuclear system interacts with the local condensed matter environment.

Our focus in recent years has been on models in which we propose that phonon exchange takes place during a nuclear reaction that occurs in a lattice. If exchange happens in the case of a highly-excited phonon mode, quantum coupling may occur with other processes that exchange phonons with the same phonon mode.

In the case of deuteron-deuteron fusion reactions, the resonating group approximation was used for many years as the primary theoretical tool with which to understand reactions, and to predict reaction cross sections. We have been interested in the generalization of the resonating group method to include lattice effects. A possible generalization is one in which the channel separation factors of the resonating group method, which describe the center-of-mass dynamics, is replaced by a more general channel separation factor which describes the center-of-mass dynamics of the reacting nuclei on the same footing with other nuclei in the lattice. We have termed this a "lattice resonating group method."

To make detailed calculations with this approach, we need to be able to compute matrix elements and interaction potentials in the presence of phonon exchange. To 
implement such calculations, one approach is to simply include phonon operators in the basic description of a nucleon coordinate. For example, if nucleon $j$ were bound to a nucleus, we would be perfectly content in a vacuum calculation thinking of the position $\mathbf{r}_{j}$ of the nucleon as being relative to the nuclear center-of-mass coordinate, and write

$$
\mathbf{r}_{j}=\mathbf{R}_{a}+\mathbf{x}_{j}
$$

where $\mathbf{R}_{a}$ is the center-of-mass coordinate of nucleus $a$, and where $\mathbf{x}_{j}$ is the position of nucleon $j$ relative to the nuclear center of mass. In the generalized problem in which the lattice is made part of the problem, the nuclear center-of-mass coordinate is a phonon operator. In this case, we would write instead

$$
\hat{\mathbf{r}}_{j}=\hat{\mathbf{R}}_{a}+\mathbf{x}_{j}
$$

with the associated interpretation that the nucleon coordinate $\hat{\mathbf{r}}_{j}$ itself depends on the lattice through the position of the nuclear center-of-mass coordinate $\hat{\mathbf{R}}_{a}$, which is an explicit phonon operator.

To compute nuclear matrix elements including phonon exchange, we need simply to express the nuclear wavefunctions as explicit functions of the spatial coordinates, and then perform integrations as required. Since the wavefunctions and the nuclear interaction have spin and isospin degrees of freedom, this task very quickly becomes much less simple. There is the additional difficulty that nuclear physicists generally make use of Racah algebra based on an independent nucleon approximation, and there does not exist much in the literature which is helpful in implementing phonon exchange more fundamentally in the case of general wavefunctions. Consequently, we have undertaken a program in which the wavefunctions are written explicitly as functions of space, spin and isospin; and in which matrix elements are reduced to sums of spatial integrals. In this paper, we focus on the four-body problem.

\section{Four-body Wavefunctions}

Group theory and the Pauli exclusion principle place rigid constraints on the form of the wavefunction. The exclusion principle dictates that the total wavefunction has to be totally antisymmetric. Now each wavefunction is characterized by a total spin and isospin. However by Schur-Weyl duality the spin (isospin) determines the irreducible representation of the symmetric group that the spin (isospin) wavefunction can belong to. The Clebsch-Gordan series of the symmetric group then constrains the possible symmetries of the spatial wavefunctions. In general, the wavefunctions are of the form

$$
\Psi=\sum_{j} C_{j}[R]_{j}[S]_{j}[T]_{j}
$$

where $[R]_{j},[S]_{j}$ and $[T]_{j}$ are the space, spin and isospin parts respectively and the $C_{j}$ are some products of the Clebsch-Gordan coefficients of the symmetric group, $S(4)$. 
In this paper, we focus on the $\mathrm{T}=0$ case only. This is the simplest case and contains the important example of $\mathrm{d}-\mathrm{d}$ fusion reactions. The generalization to other isospins is conceptually and computationally completely straightforward. However, the number of terms increases dramatically. At this level of discussion, we will neglect the Coulomb potential which does not conserve isospin.

\subsection{Basis vector labels}

The labeling of the basis vectors (in our case, it would be the space, spin or isospin wavefunctions) of any representation of the symmetric group can be done via the Yamanouchi symbols ${ }^{1}$. These symbols determine how the wavefunction (basis vector) transforms under the groups $S(2) \subset S(3) \subset S(4)$. The shorthand used for the Yamanouchi symbols is defined in Table 1.

Table 1. Shorthand for Yamanouchi Symbols

\begin{tabular}{|c|c|c|c|}
\hline Yamanouchi Symbol & Shorthand & Yamanouchi Symbol & Shorthand \\
\hline 4321 & 1 & 2121 & 6 \\
3211 & 2 & 2111 & 7 \\
3121 & 3 & 1211 & 8 \\
1321 & 4 & 1121 & 9 \\
2211 & 5 & 1111 & 10 \\
\hline
\end{tabular}

\subsection{The isospin-zero ladder wavefunctions}

Below we explicitly write down the $T=0$ ladder wavefunctions. Please note that the subscripts on the l.h.s. of the equations are merely ways of enumerating the possible wavefunctions, where as the subscripts on the r.h.s. are the shorthands for the Yamanouchi symbols (see Table 1).

$\mathbf{S}=\mathbf{0}$

$$
\begin{aligned}
& \Psi_{1}=\psi_{10} \frac{1}{\sqrt{2}}\left[s_{5} t_{6}-s_{6} t_{5}\right] \\
& \Psi_{2}=\psi_{1} \frac{1}{\sqrt{2}}\left[s_{5} t_{5}+s_{6} t_{6}\right] \\
& \Psi_{3}=-\frac{1}{2} \psi_{5}\left[s_{5} t_{6}+s_{6} t_{5}\right]-\psi_{6}\left[s_{5} t_{5}-s_{6} t_{6}\right]
\end{aligned}
$$

$\mathrm{S}=1$

$\Psi_{4}=\frac{1}{\sqrt{6}} \psi_{7}\left[s_{8} t_{6}-s_{9} t_{5}\right]+\frac{1}{2 \sqrt{3}} \psi_{8}\left[\sqrt{2} s_{7} t_{6}+s_{8} t_{6}+s_{9} t_{5}\right]+\frac{1}{2 \sqrt{3}} \psi_{9}\left[-\sqrt{2} s_{7} t_{5}+s_{8} t_{5}-s_{9} t_{6}\right]$

$\Psi_{5}=\frac{1}{2 \sqrt{3}} \psi_{2}\left[\sqrt{2} s_{7} t_{6}-s_{8} t_{6}-s_{9} t_{5}\right]-\frac{1}{2 \sqrt{3}} \psi_{3}\left[\sqrt{2} s_{7} t_{5}+s_{8} t_{5}-s_{9} t_{6}\right]+\frac{1}{\sqrt{6}} \psi_{4}\left[s_{8} t_{5}+s_{9} t_{6}\right]$

$\mathrm{S}=\mathbf{2}$

$$
\Psi_{6}=\frac{1}{\sqrt{2}}\left[\psi_{5} s_{10} t_{6}-\psi_{6} s_{10} t_{5}\right]
$$




\section{The Nuclear Potential}

We will use the Hamada-Johnston ${ }^{2}(\mathrm{H}-\mathrm{J})$ as our nuclear potential. It is one of the simplest isospin preserving nuclear potentials to give reliable results The H-J can be written down as

$$
H_{H J}=V_{C}+V_{T} S_{12}+V_{L S} \vec{L} \cdot \vec{S}+V_{L L} L_{12}
$$

where the individual potentials are defined as

$$
\begin{aligned}
V_{C} & =\vec{\tau}_{1} \cdot \vec{\tau}_{2} \vec{\sigma}_{1} \cdot \vec{\sigma}_{2} y_{C}\left(r_{12}\right) \\
V_{T} & =\vec{\tau}_{1} \cdot \vec{\tau}_{2} y_{T}\left(r_{12}\right) \\
S_{12} & =3 \frac{\left(\vec{\sigma}_{1} \cdot \vec{r}_{12}\right)\left(\vec{\sigma}_{2} \cdot \vec{r}_{12}\right)}{r_{12}^{2}}-\vec{\sigma}_{1} \cdot \vec{\sigma}_{2} \\
V_{L S} & =y_{L S}\left(r_{12}\right) \vec{L} \cdot \vec{S} \\
V_{L L} & =y_{L L}\left(r_{12}\right) \\
V_{L L} & =\vec{\sigma}_{1} \cdot \vec{\sigma}_{2} \vec{L}^{2}-\frac{1}{2} \vec{\sigma}_{1} \cdot \vec{L} \vec{\sigma}_{2} \cdot \vec{L}-\frac{1}{2} \vec{\sigma}_{2} \cdot \vec{L} \vec{\sigma}_{1} \cdot \vec{L}
\end{aligned}
$$

and where the spatial functions are given by

$$
\begin{aligned}
y_{C}^{\alpha}(x) & =0.08 \frac{\mu}{3} Y(x)\left\{1+a_{C} Y(x)+b_{C} Y^{2}(x)\right\} \\
y_{T}^{\alpha}(x) & =0.08 \frac{\mu}{3} Z(x)\left\{1+a_{T} Y(x)+b_{T} Y^{2}(x)\right\} \\
y_{L S}^{\alpha}(x) & =\mu G_{L S} Y^{2}(x)\left\{1+b_{L S} Y(x)\right\} \\
y_{L L}^{\alpha}(x) & =\mu G_{L L} x^{-2} Z(x)\left\{1+a_{L L} Y(x)+b_{L L} Y^{2}(x)\right\}
\end{aligned}
$$

and $\alpha$ stands for singlet-odd, singlet-even, triplet-odd or triplet-even.

\section{Matrix Element Calculation}

The basic idea of the calculation is straightforward. We begin with initial and final states defined as

$$
\Psi=\sum_{j} C_{j}[R]_{j}[S]_{j}[T]_{j} \quad \Psi^{\prime}=\sum_{k} C_{k}\left[R^{\prime}\right]_{k}\left[S^{\prime}\right]_{k}\left[T^{\prime}\right]_{k}
$$

we can formally calculate the matrix element to be

$$
\begin{aligned}
\left\langle\Psi\left|H_{H J}\right| \Psi^{\prime}\right\rangle & =\left\langle\sum_{j} C_{j}[R]_{j}[S]_{j}[T]_{j}\left|H_{H J}\right| \sum_{k} C_{k}^{\prime}\left[R^{\prime}\right]_{k}\left[S^{\prime}\right]_{k}\left[T^{\prime}\right]_{k}\right\rangle \\
& =\sum_{j k} C_{j}^{*} C_{k}^{\prime}\left\langle[R]_{j}\left|H_{\text {scalar }}\right|\left[R^{\prime}\right]_{k}\right\rangle
\end{aligned}
$$

where

$$
H_{\text {scalar }}=\left\langle[S]_{j}[T]_{j}\left|H_{H J}\right|\left[S^{\prime}\right]_{k}\left[T^{\prime}\right]_{k}\right\rangle
$$




\subsection{Specific result}

We have calculated a complete set of matrix elements for the $T=0$ case. There are too many cases to be presented here. We therefore focus on a specific example by calculating the tensor force matrix element of $\Psi_{1}\left(M_{s}=0\right)$ and $\Psi_{6}\left(M_{s}=1\right)$.

$$
\left\langle\Psi_{1}(0)\left|V_{T}\right| \Psi_{6}(1)\right\rangle=\int \psi_{10}^{*} \frac{3 \sqrt{3}\left(x_{12}+i y_{12}\right) z_{12} y_{T}^{e t}\left(r_{12}\right)}{r_{12}^{2}} \psi_{5} d^{3} \vec{r}_{1} \cdots d^{3} \vec{r}_{4}
$$

where $\psi_{10}^{*}=\psi_{10}^{*}\left(\vec{r}_{1}, \cdots, \vec{r}_{4}\right)$ and $\psi_{5}=\psi_{5}\left(\vec{r}_{1}, \cdots, \vec{r}_{4}\right)$ are functions of spatial coordinates alone.

\section{Transition matrix element for a $T=0$ fusion reaction}

We can now formally calculate the reaction matrix elements for the d-d fusion and phonon-coupled reactions. Since we are considering isospin-preserving reactions, we will only consider the $T=0$ case. The relevant states are

- One $4+0$ state of ${ }^{4} \mathrm{He}$ in a spin singlet ground state (mostly spatially symmetric).

- One $2+2$ state of two deuterons in a quintet spin 2 state $^{\mathrm{a}}$.

- Two $3+1$ states (since these can be singlets or triplets). These are a linear superpositions of the ${ }^{3} \mathrm{H}+p$ and ${ }^{3} \mathrm{He}+n$ states.

\subsection{Physical wavefunctions}

- The helium wavefunction is just $\Psi_{1}$ with a spatial part which is completely symmetric.

- The two deuterons to be in a spin 2 state. So

$$
\Psi_{2,2}=\mathcal{A}\left\{\psi(12 ; 34) s_{10} t_{6}\right\}
$$

where

- $\mathcal{A}$ means the antisymmetrizer

$-\psi(12 ; 34)$ is the spatial part of the deuteron wavefunctions and is of the form

$$
\psi(12 ; 34)=\phi_{d}\left(\vec{r}_{2}-\vec{r}_{1}\right) \phi_{d}\left(\vec{r}_{4}-\vec{r}_{3}\right) F\left(\frac{\vec{r}_{1}+\vec{r}_{2}}{2}, \frac{\vec{r}_{1}+\vec{r}_{2}}{2}\right)
$$

- There are two $3+1$ wavefunctions ${ }^{\mathrm{b}}$. The $S=0$

$$
\begin{aligned}
\Psi_{3,1}= & \mathcal{A}\left\{\psi(123 ; 4) \frac{1}{2}\left(s_{5} t_{121}\left(m_{T}=1 / 2\right) \downarrow_{4}-s_{6} t_{211}\left(m_{T}=1 / 2\right) \downarrow_{4}\right)\right. \\
& \left.-\psi(123 ; 4) \frac{1}{2}\left(s_{5} t_{121}\left(m_{T}=-1 / 2\right) \uparrow_{4}-s_{6} t_{211}\left(m_{T}=-1 / 2\right) \uparrow_{4}\right)\right\}
\end{aligned}
$$

\footnotetext{
${ }^{a}$ We should in general consider the deuterons to be in a singlet or triplet as well. However, we are only doing an example here. In this enumeration of states, we are ignoring the various $M_{S}$ values.

${ }^{\mathrm{b}}$ The subscripts 211, 121 and 111 are the Yamanouchi symbols for the 3-body wavefunctions
} 
and the $S=1$

$$
\begin{aligned}
\Psi_{3,1}^{\prime}= & \mathcal{A}\left\{\psi(123 ; 4) \frac{1}{2}\left(s_{8} t_{121}\left(m_{T}=1 / 2\right) \downarrow_{4}-s_{9} t_{211}\left(m_{T}=1 / 2\right) \downarrow_{4}\right)\right. \\
& \left.-\psi(123 ; 4) \frac{1}{2}\left(s_{8} t_{121}\left(m_{T}=-1 / 2\right) \uparrow_{4}-s_{9} t_{211}\left(m_{T}=-1 / 2\right) \uparrow_{4}\right)\right\}
\end{aligned}
$$

where

- the spatial part of $\Psi_{3,1}$ and $\Psi_{3,1}^{\prime}$ is of the form

$$
\psi(123 ; 4)=\phi_{3}\left(\vec{r}_{1}, \vec{r}_{2}, \vec{r}_{3}\right) F\left(\frac{\vec{r}_{1}+\vec{r}_{2}+\vec{r}_{3}}{3}, \vec{r}_{4}\right)
$$

where $\phi_{3}$ only depends on the two coordinate differences such as $\vec{r}_{1}-\vec{r}_{2}$ and $\vec{r}_{2}-\vec{r}_{3}$.

- $\uparrow_{4}$ or $\downarrow_{4}$ refer to the isospin of the fourth particle.

To compute the matrix element, we need to change basis and describe our physical wavefunctions in terms of our ladder basis.

\subsection{Physical wavefunctions in terms of ladder basis}

This change of basis can be accomplished either using induction coefficients of the symmetric group or by brute force calculations. The results are ${ }^{\mathrm{c}}$

$$
\begin{aligned}
& \mathcal{A}\left\{\Psi_{4,0}\right\}=\Psi_{1} \\
& \mathcal{A}\left\{\Psi_{2,2}\right\}=\Psi_{6}
\end{aligned}
$$

where

$$
\begin{gathered}
\psi_{5}=\frac{1}{\sqrt{12}}[2 \psi(12 ; 34)+2 \psi(34 ; 21)-\psi(23 ; 14)-\psi(14 ; 23)-\psi(13 ; 24)-\psi(24 ; 13)] \\
\psi_{6}=\frac{1}{2}[-\psi(23 ; 14)-\psi(14 ; 23)+\psi(13 ; 24)+\psi(24 ; 13)] \\
\mathcal{A}\left\{\Psi_{3,1}\right\}=\Psi_{1}
\end{gathered}
$$

where

$$
\begin{gathered}
\psi_{10}=\frac{1}{2}[\psi(123 ; 4)+\psi(124 ; 3)+\psi(134 ; 2)+\psi(234 ; 1)] \\
\mathcal{A}\left\{\Psi_{3,1}^{\prime}\right\}=\Psi_{4}
\end{gathered}
$$

${ }^{\mathrm{c}}$ The results will only involve $\Psi_{1}, \Psi_{4}$ and $\Psi_{6}$. These wavefunctions and no others are involved because of group theoretical considerations and our assumptions that the spatial part of the deuteron, triton and helium wavefunctions are symmetric under the exchange of any two particles. 
where

$$
\begin{aligned}
& \psi_{7}=\frac{1}{\sqrt{12}}[3 \psi(123 ; 4)-\psi(124 ; 3)-\psi(134 ; 2)-\psi(234 ; 1)] \\
& \psi_{8}=\frac{1}{\sqrt{6}}[4 \psi(124 ; 3)-\psi(134 ; 2)-\psi(234 ; 1)] \\
& \psi_{9}=\frac{1}{\sqrt{2}}[\psi(134 ; 2)-\psi(234 ; 1)]
\end{aligned}
$$

Now the computation of the relevant matrix elements is made simpler because we have a complete set of results for the matrix elements available in terms of the ladder basis.

\section{Matrix Elements}

We focus here on one specific example:

$$
\left\langle\mathcal{A}\left\{\Psi_{3,1}\left(M_{s}=0\right)\right\}\left|V_{T}\right| \mathcal{A}\left\{\Psi_{2,2}\left(M_{s}=1\right)\right\}\right\rangle=\left\langle\Psi_{1}\left(M_{s}=0\right)\left|V_{T}\right| \Psi_{6}\left(M_{s}=1\right)\right\rangle
$$

The rest of the matrix elements can be calculated in an exactly similar way. From our previous example we already know that

$$
\left\langle\Psi_{1}\left(M_{s}=0\right)\left|V_{t}\right| \Psi_{6}\left(M_{s}=1\right)\right\rangle=\int \psi_{10}^{*} \frac{3 \sqrt{3}\left(x_{12}+i y_{12}\right) z_{12} y_{T}^{e t}\left(r_{12}\right)}{r_{12}^{2}} \psi_{5} d^{3} \vec{r}_{1} \cdots d^{3} \vec{r}_{4}
$$

where

$$
\begin{aligned}
\psi_{5} & =\frac{1}{\sqrt{12}}[2 \psi(12 ; 34)+2 \psi(34 ; 21)-\psi(23 ; 14)-\psi(14 ; 23)-\psi(13 ; 24)-\psi(24 ; 13)] \\
\psi_{10} & =\frac{1}{2}[\psi(123 ; 4)+\psi(124 ; 3)+\psi(134 ; 2)+\psi(234 ; 1)]
\end{aligned}
$$

\section{Conclusions}

To include phonon exchange in a description of nuclear reactions, we require a description explicitly in terms of spatial wavefunctions. We have developed a complete set of such nuclear wavefunctions for the 2-body, 3-body, 4-body problems using a ladder basis. Results for the 4-body case is presented above. A complete set of matrix elements for the Hamada-Johnston potential has been developed in terms of explicit spatial integrals. Antisymmetric wavefunctions that are appropriate for deuteron-deuteron fusion calculations in the $T=0$ approximation have been developed, and we have presented a specific example in this paper.

\section{References}

1. Hamermesh, M. Group theory and its applications to physical problems, 1962. AddisonWesley.

2. Hamada, T. and Johnston, I. D. Nucl. Phys. A 34, 382. 